\title{
Reliability Analysis of Shield Tunnel Excavation Face Based on Copula Theory
}

\author{
Wen-Wei QIN ${ }^{\mathrm{a},{ }^{*}}$, Li-Mao ZHANG, Xian-Guo WU and Wei ZHANG \\ School of Civil Engineering and Mechanics, Huazhong University of Science and Technology, \\ Wuhan, Hubei 430074, China \\ awenweiqinhuster@163.com \\ ${ }^{*}$ Corresponding author
}

Keywords: Shield tunneling, Supporting pressure, Surface subsidence, Copula function, Monte Carlo method, Reliability analysis.

\begin{abstract}
Shield excavation technology is widely used in subway tunnel construction. There are complex risk factors in the process of tunnel excavation, and there is a certain correlation between them. The traditional reliability analysis is difficult to characterize the coupling relationship. In this paper, the Copula theory can accurately describe the characteristics of the correlation between parameters, and build the joint distribution function between support pressure and surface settlement. The correctness of this method is verified by engineering examples. Finally, the results show that the Frank function of Archimedean Copula with single parameter is the best Copula function. It can well characterize the joint distribution function between support pressure and surface settlement, and realize the random simulation more accurately. Combining with Monte Carlo simulation, more reasonable reliability results can be obtained. There is a strong negative correlation between the two parameters, neglecting this negative correlation will overestimate the failure probability of the parameter.
\end{abstract}

\section{Introduction}

As China's subway tunnel construction scale continues to increase, the tunnel excavation technology also tends to be more mature. The commonly used excavation methods in the project include the shield tunneling method, open-cutting tunneling method and undercutting method. Among them, the use of shield tunnel boring machine for excavation in construction projects is favored by the construction side due to its efficient construction schedule and lower cost. Although the shield method has already been used in China and has relatively mature technical support, there are still some security issues that need to be solved [1].

Recently, many experts and scholars have studied the reliability of the excavation process of shield tunnels. Wang Haitao [2] used the finite element numerical simulation method to calculate the ultimate support force of tunnel excavation face under the pre-support condition of pipe roof, and carried out the reliability analysis. In general, reliability studies often need to determine the joint probability distribution function between parameters. Therefore, it is of great significance to study the joint probability distribution of controlling factors of supporting pressure and ground settlement in the process of shield construction.

Copula theory has been widely applied in the fields of finance, insurance and hydrology domestic and foreign [3-5]. In this paper, the Copula theory can be used to accurately describe the characteristics of the correspondence between parameters without the need for large amounts of test data, and to construct a joint distribution function between support pressure and ground settlement, find the optimal Copula function for both, and use the actual The project was verified. Finally, Monte Carlo method was used to analyze the reliability of shield tunnel construction and provide reference for the actual project. 


\section{Theoretical Bases}

\section{Introduction to Copula Function Theory}

The Copula function originated from Sklar's theorem [7].

The significance of Sklar's theorem is to divide the construction process of multidimensional parameter joint distribution (JDF) into two main steps: 1) determine the optimal edge distribution function from the original data; 2) determine the optimal Copula function to characterize the parameters Function structure. The two steps can be performed independently without time requirements. Therefore, according to Sklar's theorem, the two-dimensional Copula joint distribution of two random variables $\mathrm{x}_{1}$ and $\mathrm{x}_{2}$ is as follows:

$$
F\left(x_{1}, x_{2}\right)=C\left(F_{1}\left(x_{1}\right), F_{2}\left(x_{2}\right) ; \theta\right)=C\left(\mu_{1}, \mu_{1} ; \theta\right)
$$

$C\left(\mu_{1}, \mu_{1} ; \theta\right)$ is the Copula function; $\theta$ is a function of the undetermined parameter that characterizes the correlation between $\mathrm{x}_{1}$ and $\mathrm{x}_{2}$.

Based on the research in this paper, the relationship between support pressure and ground settlement, and the initial observation of the original data, the two have a strong negative correlation. Therefore, this article selects the Copula function as shown. Among them, Gaussian is an elliptic Copula function, No.16 Copula and Frank Copula are Archimedean Copula functions, and commonly used Plackett Copula functions.

\section{Estimation of Copula Function Related Parameters}

The parameter $\boldsymbol{\theta}$ can be obtained by the Pearson correlation coefficient method and the Kendall method. The degree of dependence of two random variables can be measured by $\tau_{k}$ and has nothing to do with the marginal distribution of variables, so this method is also called nonparametric method. The non-parametric estimation of a variable $\left(\mathrm{X}_{1}, \mathrm{X}_{2}\right)$ with $\mathrm{N}$ sets of data can be calculated by equation.3:

$$
\tau_{k}=\left(\begin{array}{l}
N \\
2
\end{array}\right)^{-1} \sum_{i<j} \operatorname{sign}\left[\left(x_{1 i}-x_{1 j}\right)\left(x_{2 i}-x_{2 j}\right)\right]
$$

Here, $\operatorname{sign}(\cdot)$ is defined as:

$$
\begin{gathered}
\text { sign }=\left\{\begin{array}{ll}
1 & \left(x_{1 i}-x_{1 j}\right)\left(x_{2 i}-x_{2 j}\right) \geq 0 \\
-1 & \left(x_{1 i}-x_{1 j}\right)\left(x_{2 i}-x_{2 j}\right)<0
\end{array} \quad i, j=1,2, \ldots, N\right. \\
\tau_{k}=4 \int_{0}^{1} \int_{0}^{1} C\left(u_{1}, u_{2} ; \theta\right) d C\left(u_{1}, u_{2} ; \theta\right)-1
\end{gathered}
$$

Therefore, the Copula function related parameter $\boldsymbol{\theta}$ can be determined by solving the integral equation above.

\section{Identification of Optimal Copula Functions}

Here are two commonly used information criteria: The Akaike Information Criteria and the Bayesian Information Criteria determine the optimal Copula function. Its specific definition formula is as follows:

$$
\begin{gathered}
A I C=-2 \sum_{i=1}^{N} \ln c\left(u_{l i}, u_{2 i}\right)+2 k \\
B I C=-2 \sum_{i=1}^{N} \ln c\left(u_{l i}, u_{2 i}\right)+k \ln N
\end{gathered}
$$

Where $k$ is the number of Copula parameters, and $\mathrm{N}$ is the sample size of the related data. The 
Copula function corresponding to the minimum AIC and BIC values is the optimal Copula function. In this paper, the original observational data is transformed into $[0,1]$ uniformly distributed variables based on empirical cumulative distribution of variables.

$$
\left\{\begin{array}{l}
u_{1 i}=\frac{\operatorname{rank}\left(x_{1 i}\right)}{N+1} \\
u_{2 i}=\frac{\operatorname{rank}\left(x_{2 i}\right)}{N+1}
\end{array} \quad i=1,2, \cdots, N\right.
$$

\section{Empirical Analysis}

\section{Project Overview}

This project is a civil engineering project of the section between Dongfang Macheng Station-and Changfeng Station in Wuhan City. There are 3 curves with a radius of 450meter in the interval, and the thickness of the cover soil is 9.74-21.4meter. The radius of the tunnel is 2.75 meter.

The section of the tunnel traversing the stratum mainly consists of general cohesive soil. The upper soil layer is mainly composed of (1-1) miscellaneous soils with a thickness of 2.1 meters, (1-2) prime filling thickness of 1.5 meters, and 3-1 clay thickness of 4.5 meters. (3-2) Clay thickness 2.3 meters, (3-4) silty silty clay 4.1 meters, (3-4a) silty clay clip silt thickness 3 meters. The tunnel mainly passes through (3-2) a thickness of $0.5 \mathrm{~m}$; (3-4) a thickness of $4.1 \mathrm{~m}$; (3-4a) a thickness of 1.8 meters. The interval tunnels partially pass through the confined water layer and the elevation of the water level is about 2.2 to 3.5 meters below the ground.

\section{Identification of Copula Functions}

1. Identification and Selection of Optimal Edge Distribution Fitting Functions

This paper uses the AIC and BIC criteria to test the optimal marginal distribution functions for lognormal distribution, truncated extremum distribution, truncated normal distribution, and Weibull distribution.

Table 1 Statistics of monitoring parameters

\begin{tabular}{cccrrc}
\hline Monitoring data & Mean & SD & MAX & MIN & COV \\
\hline support pressure $(\mathrm{kpa})$ & 93.0526 & 8.9815 & 109.770 & 78.791 & 0.0965 \\
\hline surface settlement $(\mathrm{mm})$ & 15.2732 & 0.7049 & 16.760 & 14.129 & 0.0462 \\
\hline
\end{tabular}

2. Identification and selection of optimal Copula functions

For the identification of the optimal Copula function, the first is to estimate the relevant parameters of the selected Copula function based on the original data. Then calculate the AIC and BIC values of different Copula functions for support pressure and ground settlement, select the Copula function with the smallest AIC and BIC values as the optimal Copula function, and the results are shown in Table 2.

Table 2 AIC and BIC criteria calculation results

\begin{tabular}{ccccc}
\hline Copula Function & Plackett & Gaussian & No.16 & Frank \\
\hline AIC value & -96.1991 & -107.1178 & -88.3471 & -116.1950 \\
BIC value & -94.0883 & -105.0069 & -86.2363 & -114.0841 \\
Parameter value & 0.0112 & -0.9392 & 0.0018 & -16.0968 \\
\hline
\end{tabular}

Through the above analysis, the joint distribution function under the Copula theory is constructed. In order to more intuitively show the differences between different Copula functions, the simulation results of four different Copula functions are given in this paper. It can be seen that the scatter plot of the Frank function can well contain the original data. 

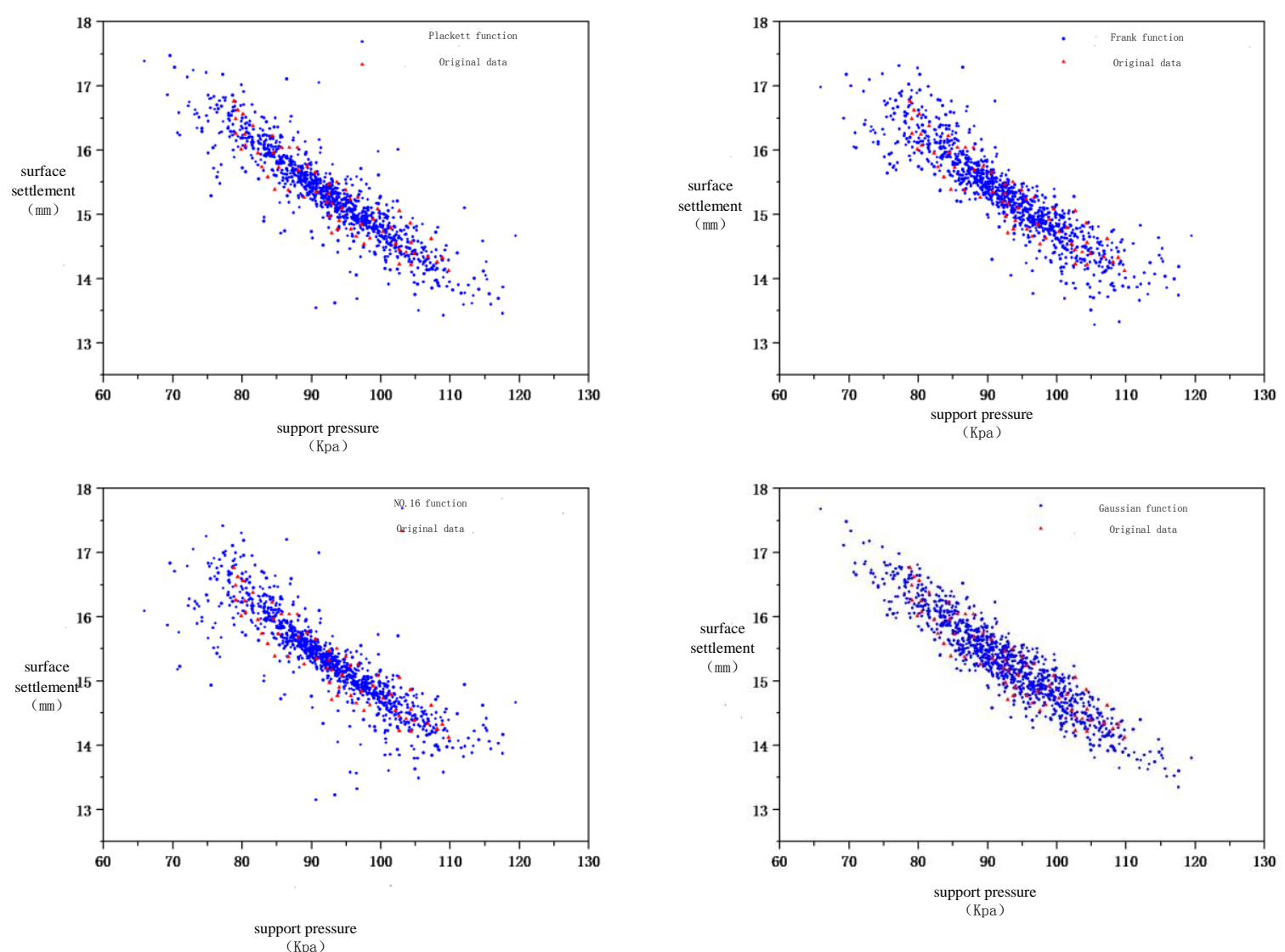

Fig. 1 Different Copula functions simulate scatter plots

\section{Shield Tunnel Construction Reliability Analysis}

\section{Functional Functions for Reliability Analysis}

In this paper, using the traditional Rankine earth pressure theory combined with the theory of limit soil balance, the passive earth pressure of the ultimate support pressure of the soil layer is calculated to be about 70Kpa; and the surface settlement caused by the construction is also required to be strictly controlled in the project. The factors, according to the project requirements, the maximum surface settlement after construction can be less than $30 \mathrm{~mm}$. The functional reliability of structural reliability is as follows:

$$
\begin{array}{r}
p_{f}=P\left[g_{1}\left(S_{1}\right)<0 \cup g_{2}\left(S_{2}\right)<0\right] \\
g_{1}\left(S_{1}\right)=R_{1}-\overline{S_{1}}, \quad g_{2}\left(S_{2}\right)=R_{2}-\overline{S_{2}}
\end{array}
$$

Among them, $P_{f}$ represents the failure probability of the system. $S_{1}$ and $S_{2}$ are the actual values of the support pressure and surface subsidence, where $\overline{S_{1}}$ and $\overline{S_{2}}$ represent the limit values for the support pressure and surface subsidence.

\section{Comparison of Reliability Results}

This model is applied to the safety and reliability assessment of the Wuhan Metro between the Macheng Station and Changfeng Station. It is planned to use this model to accurately assess the construction safety status of the shield metro area and use this result to guide the safety management of subway construction.

Using the original monitoring data, if the correlation between the parameters is not considered, but the parameters are considered to be normal distribution in the traditional way, the supporting pressure $\left(S_{1}\right)$ and surface subsidence $\left(S_{2}\right)$ scatter plot of the simulated 100000 data. As shown, the 
distribution of $S_{1}$ and $S_{2}$ is very discrete when the parameters are independent, and is evenly distributed in the coordinate system. At the same time, this paper uses Copula theory to construct a binary joint distribution model of supporting pressure and ground settlement. The Monte Carlo method can be used to obtain 100000 sets of data points for the reliability analysis of shield tunneling subway excavation construction safety.

According to the basic principle of Monte Carlo method, the failure probability value of the system is the ratio of the scattered points outside the security area to all the scattered points. In fact, there are certain dependencies between the actual parameters, which means that the traditional parameter independent model calculates the inaccuracy of the reliability results The specific calculation results are shown in Table 3.

Table 3 Different Copula Models and Their Mutual Subway Structures $p_{f}$

\begin{tabular}{cccccc}
\hline \multirow{2}{*}{ Different Copula } & \multicolumn{5}{c}{ Failure probability $p_{f}$} \\
\cline { 2 - 6 } & Plackett & Gaussian & No.16 & Frank & Independent \\
\hline & 0.0037 & 0.0023 & 0.0013 & $\mathbf{0 . 0 0 1 9}$ & 0.0051 \\
\hline
\end{tabular}

From Table 5, we can see that the pf calculated by different Copula functions is different. Since the optimal Copula function is determined by the AIC and BIC criteria as the Frank Copula function, the calculation result of the Frank Copula function is selected as the final failure probability result. This shows that the results of using dependent modeling are very different from those of independent parameters, which further validates the use of dependencies. The accuracy and necessity of sexual modeling.

\section{Summary}

(1) This paper uses the original monitoring data to construct a joint distribution function based on Copula theory for the supporting pressure and ground settlement. It is found that the Frank Copula function in Archimedean Copula function is used to fit the relationship between support pressure and ground settlement. For the optimal Copula function, the optimal edge distribution of support pressure is a normal distribution with truncated tails and an optimal edge distributed lognormal distribution with surface settlement.

(2) Using the AIC and BIC criteria, the common Gaussian Copula function is not the optimal Copula function to characterize the correlation between support pressure and ground subsidence. Moreover, different Copula functions have different reliability results. The optimal Copula function Frank Copula function can get more reasonable reliability calculation results.

(3) There is a certain negative correlation between the support pressure of earth pressure balance shield and ground settlement. If the correlation between the two is neglected for simplicity of calculation, the probability of failure in shield construction will be significantly overestimated. Caused the design to be too conservative. The selection of the Copula function type has less influence on the reliability than the correlation degree.

\section{Acknowledgement}

This research was financially supported by the National Natural Science Foundation of China(71571078, 51778262, 51708241).

\section{References}

[1] Wu Xianguo, Zeng Tiemei, Zhang Limao, et al. Study on Safety Risk Management of Adjacent Pipelines in Subway Construction [J]. Journal of Railway Engineering Society. 2013(09): 127-132.

[2] WANG Haitao, JIA Jinqing. Reliability Analysis of Tunnel Excavation Surface Stability under Condition of Pipe Shed Prestressing[J]. China Safety Science. 2011(11): 122-127. 
[3] Xiao Zhipeng, Lv Qing, Zhao Yu, et al. Analysis of Stability of Tunnel Support Based on Moving Least Square Method [J]. Journal of Wuhan University(Engineering Science). 2016(05): 683-689.

[4] Medovikov I, Prokhorov A. A New Measure of Vector Dependence, with Applications to Financial Risk and Contagion[J]. JOURNAL OF FINANCIAL ECONOMETRICS. 2017, 15(3): 474-503.

[5] Chen Y. Interplay of subexponential and dependent insurance and financial risks[J]. INSURANCE MATHEMATICS \& ECONOMICS. 2017, 77: 78-83.

[6] Yan Qixiang, Zhang Xiang. Research on joint distribution of multivariable hydrology and application of Copula function [J]. Hydroelectric Energy Science. 2010(09): 8-11.

[7] Lu Wangyong, Gao Shilong, Ma Hong. Existence theory of generalized copula[J]. Journal of Sichuan Normal University(Natural Science). 2010(02): 159-161. 